\title{
Maxillary Sinus Cholesteatoma
}

National Cancer Institute

\section{Source}

National Cancer Institute. Maxillary Sinus Cholesteatoma. NCI Thesaurus. Code C35868.

A rare, progressive, non-neoplastic pathologic process that arises from the maxillary sinus mucosal epithelium. It is characterized by the proliferation of keratinizing squamous epithelium and the formation of keratin sheets. It may lead to bone erosion and infections. Surgical removal is the appropriate treatment. 